ESAIM: PROCEEDINGS, April 2009, Vol. 26, p. 1-23

H. Ammari, Editor

\title{
AN INVERSE PROBLEM FOR FAULTS IN ELASTIC HALF SPACE
}

\author{
DARKO VOLKOV ${ }^{1}$
}

\begin{abstract}
This paper starts from a model in geophysics for the quasi static evolution of displacement fields occurring during the destabilization of fractured plates. We use the equations of linear elasticity in half space with traction free conditions on the surface and given tangential dislocations on the fault. We first discuss the derivation of the adequate Green's tensor for this problem. We then use this Green's tensor to obtain a simple and efficient approximation to the surface displacement field. Next we show how to solve the fault inverse problem from measurements of surface displacements. We first give the solution in closed form. We then illustrate it on numerical examples which demonstrate the robustness of our reconstruction algorithm.
\end{abstract}

\section{INTRODUCTION}

Faults in the Earth crust are commonly modeled as cracks in elastic half space. Stress may accumulate over long periods of time, and when some threshold is reached, plates move to a new equilibrium position. This movement may be rapid as in classical seismic events. However this can still be modeled as a quasi static process during a relatively long time interval called the nucleation phase, which precedes dynamic rupture. This was uncovered in detailed seismological observations $[3,4]$ and identified in laboratory experiments $[2,12]$. Another kind of plate movements, the so called 'silent' or 'slow' earthquakes have also been observed in nature. Accounts of silent earthquakes in subduction zones near Japan [14], New Zealand, Alaska and Mexico [8,9] were recently reported in the literature. These silent earthquakes can again be portrayed by quasi static evolution. The main practical issue that we address in this paper can be stated as a simple question: can one infer from surface displacement fields the position, depth, orientation, of a fault, and the seismic moment?

Let us now introduce a boundary value (jump) problem for a vector field modeling displacements during either a silent earthquake, or the nucleation phase of a classical earthquake. In this model the underground is assumed to be the lower half space where linear elasticity applies. Its boundary, that is the plane of equation $x_{3}=0$, will represent the Earth surface: a traction free condition will apply there. Let $\lambda>0$ and $\mu>0$ be Lamé coefficients for linear elasticity. For a displacement field $u$, we will denote the stress and strain tensors as follows,

$$
\begin{array}{r}
\sigma_{i j}(u)=\lambda \operatorname{div} u \delta_{i j}+\mu\left(\partial_{i} u_{j}+\partial_{j} u_{i}\right), \\
\epsilon_{i j}(u)=\frac{1}{2}\left(\partial_{i} u_{j}+\partial_{j} u_{i}\right) .
\end{array}
$$

${ }^{1}$ Department of Mathematical Sciences, Worcester Polytechnic Institute, Worcester MA 01609, United States; e-mail: darko@wpi.edu

(c) EDP Sciences, SMAI 2009 
and the stress vector in the normal direction $n$,

$$
T_{n}(u)=\sigma(u) n
$$

Let $\Omega$ be the lower half space $x_{3}<0$ minus a smooth, orientable, bounded surface $\Gamma$, with regular boundary $\partial \Gamma$. For simplicity, we will assume that $\Gamma$ can be contained in a plane. Let a displacement $u$ in the space $H^{1}(\Omega)^{3}$ satisfy the equations

$$
\begin{array}{r}
\mu \Delta u+(\lambda+\mu) \nabla \operatorname{div} u=0, \text { in } \Omega, \\
\sigma(u) e_{3}=0, \text { on the surface } x_{3}=0, \\
{[u \cdot n]=0,[\sigma(u) n]=0, \text { across } \Gamma,} \\
{\left[u_{\tau}\right]=g, \text { the tangential slip on } \Gamma \text { is given in } \tilde{H}^{1 / 2}(\Gamma),}
\end{array}
$$

where $\tilde{H}^{1 / 2}(\Gamma)$ is the space defined in [17]. In practice, more smoothness for $g$ is often required: $g(x)$ can be thought of decreasing such as $\rho^{1 / 2}(x)$ as $x$ approaches $\partial \Gamma$, where $\rho$ is defined on $\Gamma$ as the distance to $\partial \Gamma$. This is explained in all rigor in [17]. Condition (2) signifies that no force is applied on the surface. Condition (3) expresses the continuity of normal displacements and of the stress vector across $\Gamma$. Condition (4) gives the slip on the fault $\Gamma$, which is our only forcing term.

We will study in this paper the fault inverse problem derived from the forward problem (1-4). More precisely, given the surface displacement $u\left(x_{1}, x_{2}, 0\right)$, can the fault $\Gamma$ and the slip $g$ be recovered? This first question is of course too ambitious. Instead, we will show that it is possible to recover from the surface displacement $u\left(x_{1}, x_{2}, 0\right)$, the center of the fault $\Gamma$, a normal vector to the plane containing $\Gamma$, and the total slip $\int_{\Gamma} g$. We also propose and demonstrate on examples a robust numerical solution to the inverse problem. Note that the two dimensional analog to our present problem models the anti shear slip case, and was entirely solved in $[5,6]$.

\section{The adequate Green's tensor for the Slip on a fault in half SPaCe Problem}

In this section we compute the relevant Green's tensor $H$ for problem (1-4). This is the Green's tensor such that (1-4) may be solved by setting $u=\frac{1}{2} \int_{\Gamma} H g$, for any smooth slip $g$ on $\Gamma$.

\subsection{Past results}

If $\Omega$ is the whole space $\mathbb{R}^{3}$ it is known since Kelvin that the tensor

$$
G_{i j}(x, y)=\frac{1}{8 \pi \mu(\lambda+2 \mu)}\left((\lambda+\mu) \partial_{x_{i}} r \partial_{x_{j}} r+(\lambda+3 \mu) \delta_{i j}\right) \frac{1}{r},
$$

where $r=\sqrt{\left(x_{1}-y_{1}\right)^{2}+\left(x_{2}-y_{2}\right)^{2}+\left(x_{3}-y_{3}\right)^{2}}$, satisfies Green's problem

$$
\mu \Delta G+(\lambda+\mu) \nabla \operatorname{div} G=-I_{3} \delta_{y} \text { in } \mathbb{R}^{3} .
$$

In addition $G$ decays at infinity and has finite energy away from the singularity at $x=y$,

$$
\int_{\mathbb{R}^{3} \backslash B(y, 1)} \sigma(G(x, y)): \epsilon(G(x, y)) d x<\infty .
$$

Let $\Gamma$ be a bounded fault or cut in the space $\mathbb{R}^{3}$. Mathematically, assume that $\Gamma$ can be smoothly transformed into a disk or a polygon. The tensor $G$ can be used to express displacement fields in $\mathbb{R}^{3}$ that are continuous across $\Gamma$ and whose stress vector has a given discontinuity (sometimes called jump) across $\Gamma$.

We are interested in this paper in elastic displacement fields in the half space $x_{3}<0$, or $\mathbb{R}^{3-}$, that are traction 
free on the surface $x_{3}=0$, satisfy some discontinuity condition across a bounded surface $\Gamma$ in $\mathbb{R}^{3-}$, and decay at infinity while having finite energy. In some cases, such displacement fields $u$ can be expressed as integrals on $\Gamma$ involving a Green's tensor $M$ which satisfies

$$
\begin{array}{r}
\mu \Delta M+(\lambda+\mu) \nabla \operatorname{div} M=-I_{3} \delta_{y}, \text { in } \mathbb{R}^{3-}, \\
T_{e_{3}} M=0, \text { on the surface } x_{3}=0, \\
M \text { decays at infinity and } \int_{\mathbb{R}^{3-} \backslash B(y, 1)} \sigma(M(x, y)): \epsilon(M(x, y)) d x<\infty, \\
M(x, y)-G(x, y) \text { is smooth as } x \text { approaches } y .
\end{array}
$$

Such a Green's tensor was first computed by Mindlin, [11]. It was then re- formulated in a more compact way involving Galerkin vectors by Steketee, [16]. Sheu performed an analogous computation in the anisotropic case, see [15]. In that same paper he was able to reconstruct displacement fields produced by the 1999 Jiji, Taiwan earthquake using his new Green's tensor.

If $u$ is a finite energy elastic displacement field in the half space $x_{3}<0$, or $\mathbb{R}^{3-}$, that has zero traction on the surface $x_{3}=0$ and satisfies a stress discontinuity condition across a bounded surface $\Gamma$ in $\mathbb{R}^{3-}$, then $u$ can be expressed as the integral over $\Gamma$ of $M$ against some density that is the solution to an adequate boundary integral equation. These equations on $\Gamma$ were studied by Martin et al. in [10].

It might be costly and non trivial to solve the boundary integral equations discussed in [10]. However this can be avoided all together in some cases. Assume that we want to solve for a (finite energy, decaying at infinity) displacement field $u$ such that

$$
\begin{array}{r}
\mu \Delta u+(\lambda+\mu) \nabla \operatorname{div} u=0, \text { in } \mathbb{R}^{3-} \backslash \Gamma, \\
T_{e_{3}} u=0, \text { on the surface } x_{3}=0, \\
u \text { is continuous across } \Gamma, \\
{\left[T_{n} u\right]=f, \text { is the given jump across } \Gamma,}
\end{array}
$$

then $u$ is given by the integral formula

$$
u=\frac{1}{2} \int_{\Gamma} M f .
$$

Such a displacement field $u$ does indeed satisfy the required conditions across $\Gamma$ : this is known from potential theory and can be found in [13], at least for the free space case, and is then easily conceived in half space substituting $M$ for $G$. Let us now examine the adjoint problem to (12-15), namely, solve for a (finite energy, decaying at infinity) displacement field $u$ such that

$$
\begin{array}{r}
\mu \Delta u+(\lambda+\mu) \nabla \operatorname{div} u=0, \text { in } \mathbb{R}^{3-} \backslash \Gamma, \\
T_{e_{3}} u=0, \text { on the surface } x_{3}=0, \\
T_{n} u, \text { is continuous across } \Gamma, \\
{[u]=g, \text { is the given jump across } \Gamma .}
\end{array}
$$

We know that the analog problem in free space has the solution $u=\frac{1}{2} \int_{\Gamma}\left(T_{n(y)} G\right)^{T} g$ : this is known from potential theory and can again be found in [13]. One of our main results is the explicit computation of the Green's tensor $H$ such that problem (16-19) has the solution $u=\frac{1}{2} \int_{\Gamma} H g$, for any smooth tangential vector field on $\Gamma, g$. This highly non trivial work will appear in [18]. We merely outline in this present paper the main two steps involved in the computation. Note that some authors have incorrectly thought that $H$ could be just given by $\left(T_{n(y)} M\right)^{T}$ : this is not true for the operators $T_{e_{3}(x)}$ and $\left(T_{n(y)} \cdot\right)^{T}$ do not commute when applied to Mindlin's Green's tensor $M$. 


\subsection{Assembling Green's tensor $H$}

We start from Kelvin Green's tensor $G$ given by (5). We define a double layer potential by setting

$$
\tilde{G}(x, y, n)=\left(T_{n(y)} G(x, y)\right)^{T},
$$

\subsubsection{The image method}

The image method consists of combining $\tilde{G}(x, y, n)$ with terms from

$$
\left(T_{\bar{n}(y)} G(x, \bar{y})\right)^{T},
$$

where $\bar{n}=\left(n_{1}, n_{2},-n_{3}\right)$ and $\bar{y}=\left(y_{1}, y_{2},-y_{3}\right)$, in such a way to obtain vanishing traction on the plane $x_{3}=0$, along the $e_{1}$ and $e_{2}$ directions. More precisely, set

$$
\begin{array}{r}
\tilde{\tilde{G}}_{i j}(x, y)=\tilde{G}_{i j}(x, y, n)+\tilde{G}_{i j}(x, \bar{y}, \bar{n}), \text { for } 1 \leq i \leq 3, \quad 1 \leq j \leq 2, \\
\tilde{\tilde{G}}_{i 3}(x, y)=\tilde{G}_{i 3}(x, y, n)-\tilde{G}_{i 3}(x, \bar{y}, \bar{n}), \text { for } 1 \leq i \leq 3 .
\end{array}
$$

If $g$ is a smooth vector field on $\Gamma$ then $u(x)=\frac{1}{2} \int_{\Gamma} \tilde{\tilde{G}}(x, y) g(y)$ satisfies $(16,18,19)$ has finite elastic energy and decays at infinity. However (17) is only partially satisfied: only the first two components of $T_{e_{3}} u$ are zero at $x_{3}=0$. Therefore, we need to solve three Boussinesq problems with data

$$
-F_{j}:=\left.T_{e_{3}(x)} \tilde{\tilde{G}}_{3 j}(x, y)\right|_{x_{3}=0}, \quad j=1,2,3,
$$

to find the Green's function $H$.

\subsubsection{The Boussinesq solution in our case}

We were able to solve the three Boussinesq problems (20) in [18]. Our computational method involved working in Fourier space and switching to polar coordinates in the $x_{1}, x_{2}$ variables. Of particular importance for polar angle integration was the use of the following integrals,

$$
\int_{0}^{2 \pi} e^{i z \cos \theta} \cos p \theta d \theta=(i)^{p} 2 \pi J_{p}(z)
$$

where $p$ is an integer and $J_{p}$ is the Bessel function of the first kind of order $p$. This formula can be found in Abramowitz 9.1.21, in [1]. As to integration in radius, a formula for

$$
\int_{0}^{\infty} \frac{J_{q}(2 \pi \rho r) \rho^{p}}{\left(\rho^{2}+y_{3}^{2}\right)^{\frac{7}{2}}} d \rho, \quad p=1, . ., 5, \quad q=0, . ., 4, \quad y_{3}<0, \quad r>0,
$$

was needed. For computation of inverse Fourier transforms the following was also needed

$$
\int_{0}^{\infty} e^{2 \pi r y_{3}} J_{p}(2 \pi \rho r) r^{s} d r, \quad s=0, . ., 2, \quad p=0, . ., 4, \quad y_{3}<0, \quad \rho>0 .
$$

Closed forms for $(21,22)$, albeit intricate, can be computed. The final expression for $H$ is involved and comprises many terms. We provide it only for points on the surface $x_{3}=0$ in Appendix. 


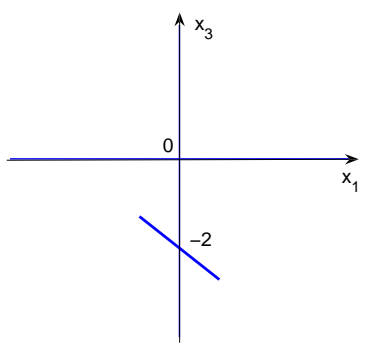

Figure $1-A$ cross section of the fault $\Gamma$ involved in the first two numerical examples. The cross section is in the plane $x_{2}=0$.

\subsection{Application: computations of displacement fields caused by a slip along a fault}

Recall that the displacement field $u$ due to a slip $g$ on a fault $\Gamma$, in the half space $x_{3}<0$, with traction free conditions on the surface $x_{3}=0$, that is problem (16-19), can be solved by setting $u=\frac{1}{2} \int_{\Gamma} H g$. We compute in this section the displacement $u$ on the surface $x_{3}=0$ using this integral formula in two examples.

These two examples involve the same geometry: a fault $\Gamma$ contained in the plane normal to the vector $(1,0,1)$, in the shape of an ellipse centered at $(0,0,-2)$. In local coordinates the ellipse has the equation ${\tilde{y_{1}}}^{2}+\left(\tilde{y_{2}} / 5\right)^{2}=1$, where local coordinates are related to the original coordinates by

$$
y=\left(\begin{array}{ccc}
s & 0 & s \\
0 & 1 & 0 \\
-s & 0 & s
\end{array}\right) \tilde{y}+\left(\begin{array}{c}
0 \\
0 \\
-2
\end{array}\right), \quad s=\frac{1}{\sqrt{2}} .
$$

A sketch of the cross section of $\Gamma$ through the plane $x_{2}=0$ appears in Figure 1.

In the first example slip occurs only in the $e_{2}$ direction. In local coordinates the slip was picked to be $g=C_{1} \sqrt{1-\tilde{y}_{1}^{2}-\left(\tilde{y_{2}} / 5\right)^{2}} e_{2}$, where the constant $C_{1}$ was adjusted in such a way that the total slip $\int_{\Gamma} g$ be of norm 1 .

In the second example, the slip does not have constant direction and is picked to be, in local coordinates, $g=C_{2}\left(-2 m^{3 / 2}, m^{1 / 2}, 2 m^{3 / 2}\right)$ where $m=1-\tilde{y}_{1}^{2}-\left(\tilde{y_{2}} / 5\right)^{2}$ and the constant $C_{2}$ was adjusted in such a way that the total slip $\int_{\Gamma} g$ be of norm 1 . 


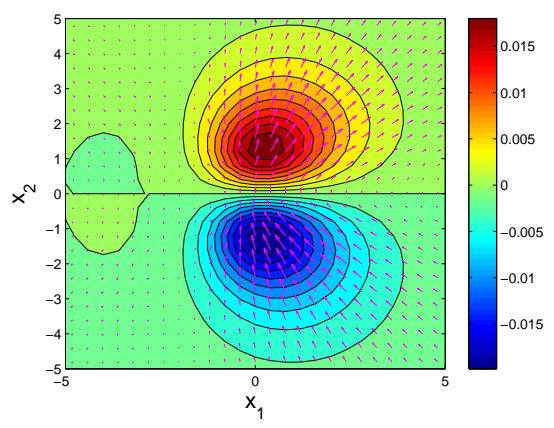

Figure 2 - Exact surface displacement for the elliptic geometry and slip in the $e_{2}$ direction considered in the first example. In local coordinates the slip was picked to be $g=C_{1} \sqrt{1-\tilde{y}_{1}^{2}-\left(\tilde{y}_{2} / 5\right)^{2}} e_{2}$, where the constant $C_{1}$ was adjusted in such a way that the total slip $\int_{\Gamma} g$ be of norm 1 . The $e_{1}$ and $e_{2}$ components of $u\left(x_{1}, x_{2}\right)$ are represented as a planar vector field using arrows, while the $e_{3}$ component is sketched on the same graph using a color contour map.

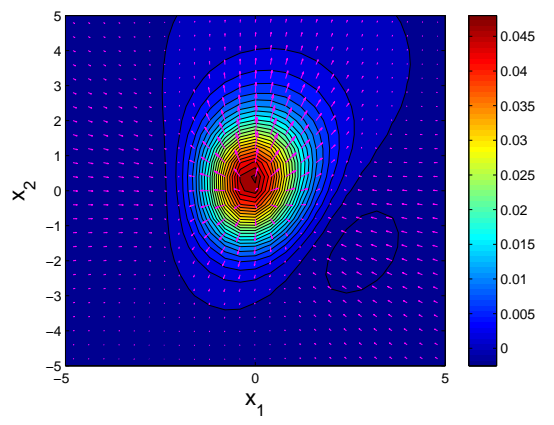

Figure 3 - Exact surface displacements in the second example. The geometry of the fault is the same as in the first example, however the slip does not now have constant direction and is picked to be, in local coordinates, $g=C_{2}\left(-2 m^{3 / 2}, m^{1 / 2}, 2 m^{3 / 2}\right)$ where $m=1-\left(\tilde{y_{1}} / 5\right)^{2}-{\tilde{y_{2}}}^{2}$ and the constant $C_{2}$ was adjusted in such a way that the total slip $\int_{\Gamma} g$ be of norm 1.

\section{Approximate SURFACE Displacements FiEldS}

Solving equations (16-19) by integrating $u=\frac{1}{2} \int_{\Gamma} H g$ might be expensive in number of operations, which is undesirable in applications where such a direct computation would have to be iterated a large number of times. We found a way to obtain a good approximate field $u\left(x_{1}, x_{2}, 0\right)$ based on asymptotics that just assume that $\left(x_{1}, x_{2}\right)$ is some distance away from the fault $\Gamma$. Suppose that the fault $\Gamma$ is centered at the point $(a, b, c)$ where $c<0$. To obtain a simpler formula for the surface displacement $u\left(x_{1}, x_{2}, 0\right)$ we now assume that either the surface point $\left(x_{1}, x_{2}\right)$ is far enough from $(a, b)$ or $|c|$ is large enough. Thus we may write

$$
\begin{array}{r}
H\left(x_{1}, x_{2}, 0, y_{1}, y_{2}, y_{3}\right)=H\left(x_{1}-y_{1}, x_{2}-y_{2}, 0,0,0, y_{3}\right)= \\
H\left(x_{1}-a, x_{2}-b, 0,0,0, c\right)+O\left(\frac{1}{\left(x_{1}^{2}+x_{2}^{2}+c^{2}\right)^{3 / 2}}\right),
\end{array}
$$


as long as $\left(y_{1}, y_{2}, y_{3}\right)$ remains on the fault $\Gamma$. ¿From there,

$$
u\left(x_{1}, x_{2}, 0\right) \simeq H\left(x_{1}-a, x_{2}-b, 0,0,0, c\right) \frac{1}{2} \int_{\Gamma} g\left(y_{1}, y_{2}, y_{3}\right) d y
$$

Setting $\left(t_{1}, t_{2}, t_{3}\right)=\frac{1}{2} \int_{\Gamma} g\left(y_{1}, y_{2}, y_{3}\right) y$, we obtain,

$$
u\left(x_{1}, x_{2}, 0\right) \simeq H\left(x_{1}-a, x_{2}-b, 0,0,0, c\right)\left(t_{1}, t_{2}, t_{3}\right) .
$$

The vector $t:=\left(t_{1}, t_{2}, t_{3}\right)$ can be interpreted as half the average slip on $\Gamma$ times the area of $\Gamma$. We will call $2 t$ the total slip on $\Gamma$.

We now proceed to demonstrate numerically the accuracy of approximation (25). We plot the relative $L^{2}$ error incurred in making the approximation (25) against depth, for three different geometries in Figure 4 . The $L^{2}$ error was computed on the surface $x_{3}=0$ in a square $[-10,10] \times[-10,10]$. In each case the fault was contained in the plane normal to the vector $(1,0,1)$ and passing through the center $(0,0, c)$, where $|c|$ is the depth, which we varied form 2 to 20 in these numerical runs. Slip was set to occur in the $e_{2}$ direction. Total slip was computed in order to apply formula (25). The plus markers correspond to a square geometry with edges of length 2 . In local coordinates $\left(\tilde{y_{1}}, \tilde{y_{2}}\right)$ centered on the fault, the slip was picked to be $\sqrt{\left(1-\left|\tilde{y_{1}}\right|\right)\left(1-\left|\tilde{y_{2}}\right|\right)}$. Local coordinates are now related to the original coordinates by

$$
y=\left(\begin{array}{ccc}
s & 0 & s \\
0 & 1 & 0 \\
-s & 0 & s
\end{array}\right) \tilde{y}+\left(\begin{array}{l}
0 \\
0 \\
c
\end{array}\right), \quad s=\frac{1}{\sqrt{2}}
$$

The star markers correspond to a circular geometry of radius 1 . In local coordinates the slip was picked to be $\sqrt{1-{\tilde{y_{1}}}^{2}-{\tilde{y_{2}}}^{2}}$.

The circle markers correspond to an elliptic geometry. The equation of the ellipse was picked to be in local coordinates, $\tilde{y}_{1}^{2}+\left(\tilde{y_{2}} / 5\right)^{2}=1$. In local coordinates the slip was picked to be $\sqrt{1-\tilde{y}_{1}^{2}-\left(\tilde{y}_{2} / 5\right)^{2}}$.

The largest error is found for the most shallow faults, that is for $|c|=2$, and ranges from $12 \%$ to $19 \%$, depending on geometry. We sketched the exact and approximated fields in the elliptic geometry case in Figures 2 and 5. It appears that the exact and approximated profiles exhibit very similar profiles.

In the remainder of the paper we will only consider surface data in the form given by the right hand side of (25). This is chiefly because this closed form formula is the basis for recovery formulas for parameters involved in the inverse problem. We will assume that fault depths will be larger than 5: note that for the fault geometries considered in this section this depth is large enough for the error in approximation (25) to be less than $4 \%$. It would be interesting to generate data for the exact field $u$ on the surface and apply our solution to the inverse problem from there. This will be done in a later paper. 


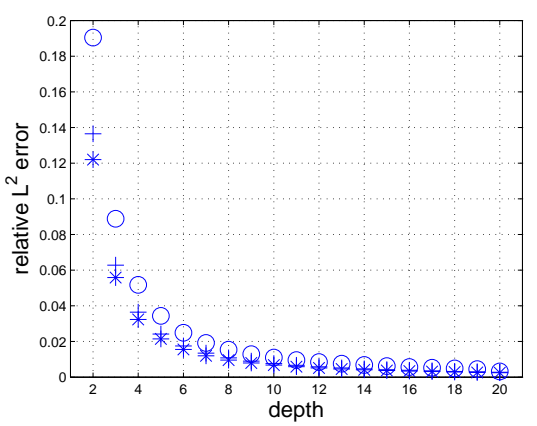

Figure 4 - The relative $L^{2}$ error incurred by making the approximation (25) plotted against depth, for three different geometries. The $L^{2}$ error was computed on the surface $x_{3}=0$ in a square $[-10,10] \times$ $[-10,10]$. In each case the fault was contained in the plane normal to the vector $(1,0,1)$ and passing through the center $(0,0, c)$, where $|c|$ is the depth. Slip was set to occur in the $e_{2}$ direction. The plus markers correspond to a square geometry with edges of length 2. In local coordinates the slip was picked to be $\sqrt{\left(1-\left|\tilde{y}_{1}\right|\right)\left(1-\left|\tilde{y}_{2}\right|\right)}$. The star markers correspond to a circular geometry with of radius 1. In local coordinates the slip was picked to be $\sqrt{1-\tilde{y}_{1}^{2}-\tilde{y}_{2}^{2}}$. The circle markers correspond to an elliptic geometry. The equation of the ellipse is in local coordinates, $\tilde{y}_{1}{ }^{2}+\left(\tilde{y_{2}} / 5\right)^{2}=1$ In local coordinates the slip was picked to be $\sqrt{1-\tilde{y}_{1}^{2}-\left(\tilde{y}_{2} / 5\right)^{2}}$.

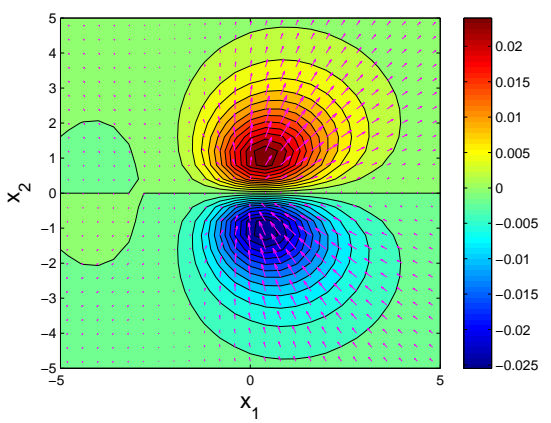

Figure 5 - Approximate surface displacement for the elliptic geometry from Figure 4 at depth 2. The approximation was obtained by applying formula (25). The computed exact field is sketched in Figure 2

\section{The FAUlt INVERSE PROBlem}

\subsection{Statement of the inverse problem and parameters to be recovered}

¿From the data $u\left(x_{1}, x_{2}\right)=H\left(x_{1}-a, x_{2}-b, 0,0,0, c\right)\left(t_{1}, t_{2}, t_{3}\right)$ introduced in (25) our goal is to find (in a numerically robust fashion):

- the center of the fault $\Gamma,(a, b, c)$

- a normal vector to the plane containing $\Gamma, n=\left(n_{1}, n_{2}, n_{3}\right)$ 
- a tangent vector to $\Gamma, t=\left(t_{1}, t_{2}, t_{3}\right)$ giving the total slip on $\Gamma$ as defined in the previous section

\subsection{Limitations}

Due to the expression for $H\left(x_{1}-a, x_{2}-b, 0,0,0, c\right)$ it turns out that $u\left(x_{1}, x_{2}\right)$ is a function that depends on $n$ and $t$ only through $s_{0}, s_{1}, s_{2}, s_{3}, s_{4}$ defined by

$$
\begin{aligned}
& s_{0}:=n_{1} t_{1}+n_{2} t_{2} \\
& s_{1}:=n_{2} t_{3}+n_{3} t_{2} \\
& s_{2}:=n_{1} t_{3}+n_{3} t_{1} \\
& s_{3}:=n_{1} t_{2}+n_{2} t_{1} \\
& s_{4}:=n_{1} t_{1}-n_{2} t_{2}
\end{aligned}
$$

Proposition 4.1. Assume that $n=\left(n_{1}, n_{2}, n_{3}\right)$ and $t=\left(t_{1}, t_{2}, t_{3}\right)$ are two orthogonal vectors in space such that $|n|=1$ and $|t| \neq 0$. Given $s_{0}, s_{1}, s_{2}, s_{3}, s_{4}$ defined in (26-30) exactly four different pairs $(n, t)$ can be reconstructed. If $(\tilde{n}, \tilde{t})$ is one reconstructed pair, the other three are $(-\tilde{n},-\tilde{t}),\left(\frac{\tilde{t}}{|\tilde{t}|}, \tilde{n}|\tilde{t}|\right)$, and $\left(-\frac{\tilde{t}}{|\hat{t}|},-\tilde{n}|\tilde{t}|\right)$.

\section{proof:}

The proof is given in [18].

\section{Proposed SOLUTion}

Denote the coordinates of the surface displacement $u_{1}\left(x_{1}, x_{2}\right), u_{2}\left(x_{1}, x_{2}\right)$, and $u_{3}\left(x_{1}, x_{2}\right)$. A calculation shows that

$$
\begin{array}{r}
I_{1}:=\iint u_{1}\left(x_{1}, x_{2}\right) d x_{1} d x_{2}=s_{2} \\
I_{2}:=\iint u_{2}\left(x_{1}, x_{2}\right) d x_{1} d x_{2}=s_{1} \\
I_{3}:=\iint u_{3}\left(x_{1}, x_{2}\right) d x_{1} d x_{2}=-\frac{1}{2} \frac{\mu}{\lambda+\mu} s_{0}
\end{array}
$$

Note that the above integrals are not in $L^{1}$ (meaning that they are not absolutely integrable). The integrand can be expanded as $\frac{A(\theta)}{\rho^{2}}+O\left(\frac{1}{\rho^{3}}\right)$, and it turns out that $\int_{0}^{2 \pi} A(\theta) d \theta=0$. The following six integrals will be needed

$$
\begin{array}{r}
I_{p, q}:=\iint u_{p}\left(x_{1}, x_{2}\right) u_{q}\left(x_{1}, x_{2}\right) d x_{1} d x_{2} \\
=\iint u_{p}\left(x_{1}+a, x_{2}+b\right) u_{q}\left(x_{1}+a, x_{2}+b\right) d x_{1} d x_{2} \\
\text { where } 1 \leq p \leq q \leq 3
\end{array}
$$

Note that the $I_{p q}$ are independent of $a$ and $b$. The $I_{p q}$ are linear combinations of $\frac{s_{i}}{c^{2}}, 0 \leq i \leq 4$ whose coefficients depend only on the Lamé constants $\lambda$ and $\mu$. We introduce six integrals that depend linearly on $a$, and six 
integrals that depend linearly on $b$.

$$
\begin{array}{r}
J_{p, q}:=\iint u_{p}\left(x_{1}, x_{2}\right) u_{q}\left(x_{1}, x_{2}\right) x_{1} d x_{1} d x_{2} \\
=\iint u_{p}\left(x_{1}+a, x_{2}+b\right) u_{q}\left(x_{1}+a, x_{2}+b\right) x_{1} d x_{1} d x_{2}+a I_{p, q} \\
K_{p, q}:=\iint u_{p}\left(x_{1}, x_{2}\right) u_{q}\left(x_{1}, x_{2}\right) x_{2} d x_{1} d x_{2} \\
=\iint u_{p}\left(x_{1}+a, x_{2}+b\right) u_{q}\left(x_{1}+a, x_{2}+b\right) x_{2} d x_{1} d x_{2}+b I_{p, q}
\end{array}
$$

note that $J_{p q}-a I_{p q}$ and $K_{p q}-b I_{p q}$ do not depend on $a$ and $b$ and are linear combinations of $\frac{s_{i}}{c^{2}}, 0 \leq i \leq 4$ whose coefficients depend only on the Lamé constants $\lambda$ and $\mu$.

Equations (31-32) provide $s_{0}, s_{1}, s_{2}$. There remains to solve for $s_{3}, s_{4}, a, b, c$, which we proceed to do next. In some cases we will find expressions for $s_{3}^{2}, s_{4}^{2}$ and quadratic equations for $c^{2}$. The solution will have to be picked, depending on the case, within a set of 1,2 , or 4 candidates. In practice making such a final pick is not costly, as we will retain the solution that leads to a reconstructed surface displacement field closest to the surface data.

Remark The integrals $I_{p}, I_{p, q}, J_{p, q}, K_{p, q}$, can be computed in a closed form. Due to the complexity of the expression for $u\left(x_{1}, x_{2}\right)$ this can only be done using a symbolic calculation software. For brevity, we do not provide here the resulting expressions. Instead we indicate how those can be used to solve the fault inverse problem.

\subsection{The case $s_{0} \neq 0$}

As $n$ and $t$ are perpendicular this is equivalent to the condition $n_{3} t_{3} \neq 0$. Physically, this means that the fault is not vertical and that the average slip is not horizontal.

\subsubsection{Solving for the depth $|c|$}

If

$$
\frac{\lambda}{\mu} \neq \frac{-1+\sqrt{10}}{3},
$$

the depth $c$ satisfies the equation

$$
A_{1} c^{4}+A_{2} c^{2}+A_{3}=0
$$

where we set

$$
\begin{array}{r}
A_{1}:=\frac{1}{2} \frac{\left(C_{3}+C_{4}\right)\left(I_{1,1}-I_{2,2}\right)^{2}}{s_{0}^{2}\left(2 C_{1}-C_{2}\right)^{2}}+C_{4} \frac{I_{1,2}{ }^{2}}{s_{0}^{2}\left(C_{1}-C_{6}\right)^{2}}, \\
A_{2}:=-I_{1,1}-I_{2,2}-\frac{C_{1}\left(C_{3}+C_{4}\right)\left(s_{2}{ }^{2}-s_{1}{ }^{2}\right)\left(I_{1,1}-I_{2,2}\right)}{s_{0}^{2}\left(2 C_{1}-C_{2}\right)^{2}} \\
-2 C_{4} \frac{C_{1} s_{1} s_{2} I_{1,2}}{s_{0}^{2}\left(C_{1}-C_{6}\right)^{2}}, \\
A_{3}:=2 C_{1}\left(s_{2}^{2}+s_{1}^{2}+2 s_{0}^{2}\right)+\frac{1}{2}\left(C_{3}-C_{4}\right) s_{0}^{2}+\frac{1}{2}\left(C_{3}+C_{4}\right) \frac{\left(C_{1}\left(s_{2}^{2}-s_{1}^{2}\right)\right)^{2}}{s_{0}^{2}\left(2 C_{1}-C_{2}\right)^{2}} \\
+C_{4} \frac{C_{1}^{2} s_{1}^{2} s_{2}{ }^{2}}{s_{0}^{2}\left(C_{1}-C_{6}\right)^{2}},
\end{array}
$$


where $C_{2}, C_{2}, C_{4}, C_{6}$ depend only on $\lambda$ and $\mu$. It can be shown that due to (36)

$$
\begin{array}{r}
2 C_{1}-C_{2} \neq 0 \\
C_{1}-C_{6} \neq 0
\end{array}
$$

It can also be shown that $I_{1,1}+I_{2,2}>0$, therefore we can not have $A_{1}=A_{2}=0$ : equation (37) is well posed.

Assume now that

$$
\frac{\lambda}{\mu}=\frac{-1+\sqrt{10}}{3}
$$

or equivalently $2 C_{1}-C_{2}=C_{1}-C_{6}=0$. We find the following relations

$$
\begin{array}{r}
\left(I_{1,1}-I_{2,2}\right) c^{2}=C_{1}\left(s_{2}^{2}-s_{1}^{2}\right) \\
I_{1,2} c^{2}=C_{1} s_{1} s_{2}
\end{array}
$$

It follows from (40)that $C_{1} \neq 0$. If $s_{1}=s_{2}=0, c$ can be solved as indicated in case 5.1.4. Otherwise $c$ can be solved by application of (41) or (42).

After solving for $c$ we proceed to solve for $s_{3}$ and $s_{4}$

5.1.2. Case $s_{0} \neq 0$ and $s_{1} \neq 0$

The following formulas hold

$$
\begin{gathered}
s_{3}=-\frac{-\left(I_{1,3} s_{1}+I_{2,3} s_{2}\right) c^{2}+\left(-C_{1}-C_{10}-C_{11}\right) s_{2} s_{1} s_{0}}{C_{1}\left(s_{1}^{2}+s_{2}^{2}\right)} \\
s_{4}=-\frac{-c^{2} I_{2,3}+1 / 2\left(-C_{1}-C_{10}-C_{11}\right) s_{1} s_{0}+C_{1} s_{3} s_{2}}{1 / 2\left(-C_{1}+C_{10}-C_{11}\right) s_{1}}
\end{gathered}
$$

Our calculations show that $\left(C_{1}-C_{10}+C_{11}\right)=\frac{3}{32 \pi}$, for all values of $\lambda$ and $\mu$. We can now apply section 4.2 to solve for the vectors $n$ and $t$.

\subsubsection{Case $s_{0} \neq 0$ and $s_{2} \neq 0$}

We can still use formula (43), and we use the following in place of (44)

$$
s_{4}=-\frac{-c^{2} I_{1,3}+1 / 2\left(-C_{1}-C_{10}-C_{11}\right) s_{2} s_{0}+C_{1} s_{3} s_{1}}{1 / 2\left(-C_{1}+C_{10}-C_{11}\right) s_{2}}
$$

5.1.4. Case $s_{0} \neq 0$ and $s_{1}=s_{2}=0$

In that case, $c, s_{3}, s_{4}$ can be solved for by using the following, assuming on the one hand (36),

$$
\begin{array}{r}
c^{2}=\frac{\left(4 C_{1}+C_{7}\right) s_{0}^{2}}{I_{3,3}} \\
s_{4}=-\frac{\left(-I_{1,1}+I_{2,2}\right) c^{2}}{\left(2 C_{1}-C_{2}\right) s_{0}} \\
s_{3}^{2}=\left(s_{0}^{2}-s_{4}^{2}\right)
\end{array}
$$


Assuming on the other hand that (40) holds we solve the equations

$$
\begin{array}{r}
c^{2}=\frac{\left(4 C_{1}+C_{7}\right) s_{0}^{2}}{I_{3,3}} \\
C_{4} s_{3}^{2}+\frac{1}{2}\left(C_{3}+C_{4}\right) s_{4}^{2}=-\left(4 C_{1}+\frac{1}{2} C_{3}-\frac{1}{2} C_{4}\right) s_{0}^{2}+2 c^{2} I_{1,1} \\
s_{3}^{2}+s_{4}^{2}=s_{0}^{2}
\end{array}
$$

where equations (50-51) form a non singular linear system in $s_{3}^{2}$ and $s_{4}^{2}$.

5.1.5. Case $s_{0} \neq 0$ : solving for the horizontal position $(a, b)$

There remains one task: solving for the horizontal position $(a, b)$. This is now straightforward by application of $(34)$ and $(35)$ for $(p, q)$ equal to $(1,1)$, or $(2,2)$, or $(3,3)$. This can be done because $I_{1,1}, I_{2,2}, I_{3,3}$ to be simultaneously zero.

\subsection{Case $s_{0}=0$}

This case is very different. Note that as $n$ and $t$ are orthogonal $s_{0}=-n_{3} t_{3}$. Physically, this case corresponds to either vertical faults or horizontal total slips (or both). Note that $s_{0}=s_{1}=s_{2}=0$ if and only if $n_{3}=t_{3}=0$.

5.2.1. Case $s_{0}=0$ and $s_{1} \neq 0$ or $s_{2} \neq 0$

In that case, either $s_{1}^{2}-s_{2}^{2} \neq 0$ or $s_{1} s_{2} \neq 0$.

If $s_{1}^{2}-s_{2}^{2} \neq 0$ then, as in that case $I_{1,1}-I_{2,2} \neq 0$

$$
\begin{array}{r}
c^{2}=C_{1} \frac{s_{2}^{2}-s_{1}^{2}}{I_{1,1}-I_{2,2}} \\
s_{3}=-\frac{-\left(I_{1,3} s_{1}+I_{2,3} s_{2}\right) c^{2}}{C_{1}\left(s_{1}^{2}+s_{2}^{2}\right)} \\
s_{4}=\frac{-\left(-I_{1,3} s_{2}+I_{2,3} s_{1}\right) c^{2}}{-\frac{1}{2}\left(-C_{1}+C_{10}-C_{11}\right)\left(s_{2}^{2}+s_{1}^{2}\right)}
\end{array}
$$

where we recall that $\left(C_{1}-C_{10}+C_{11}\right)=\frac{3}{32 \pi}$, for all values of $\lambda$ and $\mu$. If $s_{1} s_{2} \neq 0$, as $I_{1,2} \neq 0$,

$$
\begin{array}{r}
c^{2}=\frac{C_{1} s_{1} s_{2}}{I_{1,2}} \\
s_{3}=-\frac{-\left(I_{1,3} s_{1}+I_{2,3} s_{2}\right) c^{2}}{C_{1}\left(s_{1}^{2}+s_{2}^{2}\right)} \\
s_{4}=\frac{-\left(-I_{1,3} s_{2}+I_{2,3} s_{1}\right) c^{2}}{-\frac{1}{2}\left(-C_{1}+C_{10}-C_{11}\right)\left(s_{2}^{2}+s_{1}^{2}\right)}
\end{array}
$$

There remains one task: solving for the horizontal position $(a, b)$. This is done by application of (34) and (35) for $(p, q)$ equal to $(1,1)$, or $(2,2)$, or $(3,3)$.

5.2.2. Case $s_{0}=0$ and $s_{1}=s_{2}=0$

We have in this case $n_{3}=t_{3}=0$. Physically, the fault is vertical and the total slip is occurring in a horizontal direction. In this case, we begin by solving for $a$ and $b$ as we can show that

$$
a=\frac{J_{p, p}}{I_{p, p}} \text { and } b=\frac{K_{p, p}}{I_{p, p}}
$$


for $p$ being either 1,2 , or 3 .

If

$$
\frac{\lambda}{\mu} \neq \frac{-1+\sqrt{46}}{9}
$$

$c$ is easily solved for too as

$$
c=-\frac{C_{4}}{C_{13}} \frac{K_{2,3}}{I_{1,1}}
$$

where $C_{13} \neq 0$ due to (58). To solve for $s_{3}$ and $s_{4}$, we set

$$
I_{2,3}^{\prime}=\iint_{x_{2}>b} u_{2}\left(x_{1}, x_{2}\right) u_{3}\left(x_{1}, x_{2}\right) d x_{1} d x_{2}
$$

It follows that

$$
\begin{array}{r}
s_{3}^{2}=\frac{-4 I_{2,3}^{\prime} c^{2}+A\left(2 C_{16}+C_{17}\right)}{C_{17}-2 C_{16}} \\
s_{4}^{2}=-4 \frac{C_{16} A-I_{2,3}^{\prime} c^{2}}{C_{17}-2 C_{16}},
\end{array}
$$

where $A=\frac{2 c^{2} I_{1,1}}{C_{4}}$, and where it can be shown that $C_{17}-2 C_{16}>0$, for all positive values of $\lambda$ and $\mu$. If

$$
\frac{\lambda}{\mu}=\frac{-1+\sqrt{46}}{9},
$$

the above solution is not valid as $C_{13}=0$. We then introduce

$$
\begin{array}{r}
I_{1,3}^{\prime}=\iint_{x_{2}>b} u_{1}\left(x_{1}, x_{2}\right) u_{3}\left(x_{1}, x_{2}\right) d x_{1} d x_{2} \\
J_{1,3}^{\prime}=\iint_{x_{2}>b} u_{1}\left(x_{1}, x_{2}\right) u_{3}\left(x_{1}, x_{2}\right)\left(x_{1}+a\right) d x_{1} d x_{2}
\end{array}
$$

which are involved in the following equations

$$
\begin{array}{r}
c^{2} I_{1,1}=C_{20} s_{3}^{2} \\
c^{2} I_{1,3}^{\prime}=C_{21} \frac{s_{3} s_{4}}{2} \\
c J_{1,3}^{\prime}=C_{22} \frac{s_{3} s_{4}}{2}
\end{array}
$$

where the constants $C_{20}, C_{21}, C_{22}$ are all non zero. Assume, that $s_{3} s_{4} \neq 0$. Then $c$ is obtained by dividing (63) by (64), from where we can solve for $s_{3}^{2}$ and $s_{4}^{2}$.

Assume now that $s_{3} s_{4}=0$. Note that $s_{3}=0$ if and only if $n_{1}^{2}=n_{2}^{2}=\frac{1}{2}$, and $s_{4}=0$ if and only if $n_{1} n_{2}=0$. In either case we rotate the surface coordinate unit vectors $e_{1}, e_{2}$ by the angle $\frac{\pi}{8}$. In the new coordinates system, we still have $n_{3}=t_{3}=0$, but this time $s_{3} s_{4} \neq 0$. 


\section{NUMERICAL RECONSTRUCTION}

\subsection{Using formulas derived in the previous section}

We now apply the reconstruction formulas derived in the previous section. To limit the number of runs presented in this paper and to facilitate comparison between these runs, we fix $\lambda=\mu=1, a=-10, b=10$. We then consider different depths $c$, different geometries (or rather fault orientation characterized by $n$ ) and total slips given by $t$. All considered cases are indicated in the first column of table 1 . In each case we compute values for $u\left(x_{1}, x_{2}\right)$, as defined in the previous section, where $\left(x_{1}, x_{2}\right)$ is on a discrete grid of points defined by the square $[-M, M] \times[-M, M], M=200$, and the spacing $h=2$. The integrals $I_{p}, I_{p, q}, J_{p, q}, K_{p, q}$ were evaluated numerically from the discrete set of values for $\left(x_{1}, x_{2}\right)$ by application of a simple order two quadrature rule combined with estimates of tails. We then applied formulas from section 5 to reconstruct $(a, b, c), n, t$. Solutions are reported in the second column of table 1 . Note that in each case there are truly four equivalent solutions for $n, t$. We retained the one that makes for the easiest comparison to the data, for convenience.

We then found out that it is possible to sharpen our numerical solutions by applying a least square minimization technique. The minimization uses the discrete data $u\left(x_{1}, x_{2}\right)$. The approximate solution provided in the second column of table 1 serves as a starting point for the minimization. The improved solution after minimization is borne out in the third column of table 1. Note that if minimization is started from a point too far away from the true values for $(a, b, c), n, t$, it is, in most cases, unsuccessful: this underscores the importance of inversion techniques developed in section 5 .

The results obtained in the first case considered in table 1 may seem not as accurate when compared to results from other cases. In fact accuracy can be greatly improved in the first case for a smaller spacing $h$.

\subsection{Requirements on grids of data points}

We inspected which are the smallest value for $M$ and the largest value for $h$ that will guarantee success of our numerical inversion for a given center $(a, b, c)$ and any $n, t$ where the discrete data $u\left(x_{1}, x_{2}\right)$ is on the grid defined by the square $[-M, M] \times[-M, M]$, and the spacing $h$. It is in fact sufficient to carry out the inspection for $a=0, b=0, c=-1$ and to then infer all other cases by rescaling and horizontal translation. It was found that those smallest value for $M$ and the largest value for $h$ are (approximately)

$$
M_{\min }=\alpha|c|+|a|+|b|, \quad h_{\max }=\frac{5|c|}{\alpha}
$$

where

$$
2 \leq \alpha \leq 4
$$

For example if $a=b=0$ we obtain a grid of 11 by 11 points. Smaller values for $\alpha$ make for a grid that is too concentrated near the fault ( $M$ is too small). Larger values for $\alpha$ do not give fine enough details near the fault ( $h$ is too large). 


\begin{tabular}{|c|c|c|c|c|c|c|c|c|}
\hline $\begin{array}{l}a \\
n_{1} \\
t_{1} \\
\end{array}$ & $\begin{array}{l}b \\
n_{2} \\
t_{2} \\
\end{array}$ & $\begin{array}{l}c \\
n_{3} \\
t_{3} \\
\end{array}$ & & & & & & \\
\hline-10 & 10 & -5 & -11.4598 & 8.5814 & -5.7761 & -10.0242 & 9.9558 & -5.0492 \\
\hline 0.8660 & 0 & 0.5000 & 0.9144 & 0.0529 & 0.4014 & 0.8694 & 0.0154 & 0.4939 \\
\hline-1.0000 & 2.0000 & 1.7321 & -0.8932 & 2.9833 & 1.6413 & -1.0107 & 2.0236 & 1.7161 \\
\hline-10 & 10 & -10 & -12.9798 & 7.1005 & -11.5655 & -9.9819 & 9.9614 & -10.0335 \\
\hline 0.8660 & 0 & 0.5000 & 0.9157 & 0.0541 & 0.3982 & 0.8669 & 0.0100 & 0.4983 \\
\hline-1.0000 & 2.0000 & 1.7321 & -0.8884 & 3.0055 & 1.6353 & -1.0217 & 2.0025 & 1.7374 \\
\hline-10 & 10 & -40 & -16.7442 & 2.4340 & -44.0588 & -9.9501 & 9.9795 & -40.0129 \\
\hline 0.8660 & 0 & 0.5000 & 0.8997 & 0.0412 & 0.4345 & 0.8664 & 0.0041 & 0.4993 \\
\hline-1.0000 & 2.0000 & 1.7321 & -0.9242 & 2.6311 & 1.6639 & -1.0080 & 1.9955 & 1.7327 \\
\hline-10 & 10 & $\begin{array}{l}-5 \\
\end{array}$ & -10.0000 & 10.0030 & -5.0000 & -9.9994 & 10.0021 & -4.9978 \\
\hline 0.8660 & 0 & 0.5000 & 0.8657 & -0.0008 & 0.5005 & 0.8660 & -0.0006 & 0.5000 \\
\hline 0 & 2.0000 & 0 & 0.0016 & 1.9993 & 0.0004 & 0.0009 & 1.9989 & 0.0007 \\
\hline-10 & 10 & -10 & -10.0229 & 10.0077 & -10.0288 & -10.0010 & 10.0030 & -10.0018 \\
\hline 0.8660 & 0 & 0.5000 & 0.8660 & -0.0007 & 0.5001 & 0.8659 & -0.0002 & 0.5002 \\
\hline 0 & 2.0000 & 0 & 0.0015 & 2.0061 & 0.0003 & 0.0004 & 2.0001 & 0.0004 \\
\hline-10 & 10 & -40 & -10.4012 & 9.8320 & -40.1008 & -9.9919 & 10.0081 & -39.9959 \\
\hline 0.8660 & 0 & 0.5000 & 0.8625 & -0 & 0.5061 & 0.8660 & -0.0004 & 0.5001 \\
\hline 0 & 2.0000 & 0 & 0.0017 & 2.0001 & 0.0077 & 0.0013 & 1.9988 & -0.0006 \\
\hline-10 & 810 & -5 & -10.0001 & 10.0001 & -5.0118 & -9.9999 & 10.0000 & -4.9999 \\
\hline 0.7071 & 0.7071 & 0 & 0.7071 & 0.7071 & 0.0000 & 0.7071 & 0.7071 & -0.0000 \\
\hline 0 & 0 & 2.0000 & -0.0000 & 0.0000 & 2.0044 & -0.0000 & 0.0000 & 2.0000 \\
\hline-10 & 10 & -10 & -10.0015 & 10.0015 & -10.0435 & -10.0000 & 9.9999 & -10.0000 \\
\hline 0.7071 & 0.7071 & 0 & 0.7071 & 0.7071 & 0 & 0.7071 & 0.7071 & 0.0000 \\
\hline 0 & 0 & 2.0000 & -0.0000 & 0.0000 & 2.0088 & -0.0000 & 0.0000 & 2.0000 \\
\hline-10 & 10 & -40 & -10.0509 & 10.0509 & -40.2883 & -10.0000 & 9.9996 & -39.9993 \\
\hline 0.7071 & 0.7071 & 0 & 0.7071 & 0.7071 & -0 & 0.7071 & 0.7071 & 0.0000 \\
\hline 0 & 0 & 2.0000 & -0.0038 & 0.0038 & 2.0291 & -0.0000 & 0.0000 & 2.0000 \\
\hline-10 & 10 & -5 & -10.0024 & 10.0000 & -4.8745 & -10.0009 & 10.0084 & -4.9986 \\
\hline 0.7071 & 0.7071 & 0 & 0.7071 & 0.7071 & -0.0000 & 0.7071 & 0.7071 & 0.0015 \\
\hline 2.0000 & -2.0000 & 0 & 2.0144 & -2.0144 & -0.0064 & 2.0030 & -2.0029 & 0.0043 \\
\hline-10 & 10 & -10 & -10.0082 & 10.0002 & -9.5237 & -9.9991 & 10.0002 & -9.9956 \\
\hline 0.7071 & 0.7071 & 0 & 0.7071 & 0.7071 & -0.0026 & 0.7071 & 0.7072 & 0.0005 \\
\hline 2.0000 & -2.0000 & 0 & 1.9072 & -1.9072 & 0.0000 & 2.0002 & -1.9999 & -0.0009 \\
\hline-10 & 10 & -40 & -9.0590 & 10.0016 & -56.8499 & -9.9894 & 10.0500 & -40.0016 \\
\hline 0.7071 & 0.7071 & 0 & -0.9099 & -0.4149 & -0.0003 & 0.7063 & 0.7080 & -0.0007 \\
\hline 2.0000 & -2.0000 & 0 & 1.6529 & -3.6253 & -0.0031 & 2.0036 & -1.9988 & 0.0011 \\
\hline
\end{tabular}

Table 1 - Reconstruction of faults defined by $(a, b, c), n, t$. First column indicates specific values in each case. In all cases $\lambda=\mu=1$, and values for $u\left(x_{1}, x_{2}\right)$ are computed for $\left(x_{1}, x_{2}\right)$ on a discrete grid of points defined by the square $[-M, M] \times[-M, M], M=200$, and the spacing $h=2$. Second column indicates reconstruction after application of formulas derived in section 5 . Third column contains sharper results obtained by applying a least square minimization technique with results from column 2 used as starting points. 


\section{Detecting active faults from GPS observations}

The aim of this section is to account for the sensitivity of measuring apparatus and for the observation grid stepsize, and to understand how those may affect detection of active faults. In an attempt to model real life situations we choose a threshold under which surface displacements cannot be measured. Moreover, we allow ourselves to use only a limited number of measurement points, on a fixed grid, independently of the geometry and the size of the fault, and location of the center of the fault.

\subsection{Sensitivity and indicative points} if

By definition, at fixed sensitivity $S$, a point $\left(x_{1}, x_{2}\right)$ on the grid of measurement points is said to be indicative

$$
\left|u\left(x_{1}, x_{2}\right)\right| \geq S
$$

where $u\left(x_{1}, x_{2}\right)$ is the surface displacement generated by a given slip on the fault $\Gamma$. Next, at fixed sensitivity $S$, we define the indicative set $I$ to be

$$
I:=\left\{\left(x_{1}, x_{2}\right) \in \mathbb{R}^{2} ;\left|u\left(x_{1}, x_{2}\right)\right| \geq S\right\}
$$

\subsection{Fault recovery at fixed observation sensitivity}

We now seek to evaluate how the sensitivity of observations affect our inversion techniques. We know from the previous subsection that sensitivity is related to the stepsize of the grid.

We apply the reconstruction formulas derived in section 5 , while taking into account a sensitivity threshold $S$. We repeat some of the runs from table 1 at sensitivity level $S=2 \cdot 10^{-5}$ for all cases with one exception: this high sensitivity level led to failure of the recovery method for the case $c=-40 n=(0.8660,0,0.5000), t=$ $(-1.0000,2.0000,1.7321)$. We made a second run in that case with the sensitivity $S=10^{-5}$ which led to a successful recovery. All outputs of successful runs appear in table 2. In each case we plotted the region of $[-M, M] \times[-M, M], M=200$ comprised of indicative points (points where $|u| \geq S$ ). These plots appear in Figure 6 .

Finally we present a reconstruction calculation in case of scarce data, $M=M_{\min }, h=h_{\max }$, (see formula (65)), at sensitivity $S=4 \cdot 10^{-5}$. The values for $n$ and $t$ for this run are indicated in Table 3 . With those values for $M, h, S$, the number of indicative points is small. The grid of indicative points is sketched in Figure 7 . We also plotted the planar vector field $\left(u_{1}, u_{2}\right)$ at the indicative points in Figure 7 , the third component $u_{3}$ appears as a contour colored map on the same graph. 


\begin{tabular}{|lll|lll|lll|}
\hline$a$ & $b$ & $c$ & & & & & & \\
$n_{1}$ & $n_{2}$ & $n_{3}$ & & & & & & \\
$t_{1}$ & $t_{2}$ & $t_{3}$ & & & & & & \\
\hline \hline-10 & 10 & -5 & -9.3626 & 10.4380 & -5.4635 & -10.0431 & 10.0359 & -5.1183 \\
0.8660 & 0 & 0.5000 & 0.8067 & -0.0622 & 0.5877 & 0.8600 & -0.0130 & 0.5102 \\
-1.0000 & 2.0000 & 1.7321 & -1.5165 & 1.8331 & 2.2752 & -1.0468 & 2.0037 & 1.8154 \\
\hline-10 & 10 & -10 & -3.8306 & 12.8072 & -9.6300 & -9.9316 & 10.0195 & -9.9248 \\
0.8660 & 0 & 0.5000 & 0.8077 & 0.1184 & 0.5776 & 0.8650 & 0.0023 & 0.5018 \\
-1.0000 & 2.0000 & 1.7321 & -1.9958 & 0.6539 & 2.6566 & -0.9971 & 1.9754 & 1.7096 \\
\hline-10 & 10 & -40 & 28.0423 & 29.8760 & -32.0114 & -9.9138 & 9.9576 & -39.9913 \\
0.8660 & 0 & 0.5000 & -0.7673 & -0.2060 & -0.6073 & 0.8669 & 0.0107 & 0.4984 \\
-1.0000 & 2.0000 & 1.7321 & 2.4998 & 0.0825 & -3.1867 & -1.0240 & 1.9724 & 1.7390 \\
\hline \hline-10 & 10 & -5 & -10.0000 & 10.0000 & -4.3542 & -9.9999 & 10.0003 & -5.0001 \\
0.7071 & 0.7071 & 0 & 0.7071 & 0.7071 & 0.0000 & 0.7071 & 0.7071 & 0.0000 \\
0 & 0 & 2.0000 & -0.0000 & -0.0000 & 1.7412 & -0.0000 & -0.0000 & 2.0001 \\
\hline-10 & 10 & -10 & -10.0000 & 10.0000 & -7.9563 & -10.0000 & 10.0000 & -10.0000 \\
0.7071 & 0.7071 & 0 & 0.7071 & 0.7071 & 0.0000 & 0.7071 & 0.7071 & 0.0000 \\
0 & 0 & 2.0000 & 0.0000 & -0.0000 & 1.5903 & -0.0000 & -0.0000 & 2.0000 \\
\hline-10 & 10 & -40 & -10.1213 & 10.1213 & -19.3468 & -10.0003 & 10.0005 & -39.9993 \\
0.7071 & 0.7071 & 0 & -0.7071 & -0.7071 & 0.0000 & 0.7071 & 0.7071 & -0.0000 \\
0 & 0 & 2.0000 & 0.0012 & -0.0012 & -0.9467 & 0.0001 & -0.0001 & 2.0000 \\
\hline \hline
\end{tabular}

Table 2 - Reconstruction of faults defined by $(a, b, c), n, t$, at sensitivity $S=2 \cdot 10^{-5}$, except in the third case where sensitivity had to be lowered to $S=10^{-5}$. First column indicates specific values in each case. In all cases $\lambda=\mu=1$, and values for $u\left(x_{1}, x_{2}\right)$ are computed for $\left(x_{1}, x_{2}\right)$ on a discrete grid of points defined by the square $[-M, M] \times[-M, M], M=200$, and the spacing $h=2$. Second column indicates reconstruction after application of formulas derived in section 5. Third column contains sharper results obtained by applying a least square minimization technique with results from column two as initial values.

\begin{tabular}{|lll|lll|lll|}
\hline$a$ & $b$ & $c$ & & & & & & \\
$n_{1}$ & $n_{2}$ & $n_{3}$ & & & & & & \\
$t_{1}$ & $t_{2}$ & $t_{3}$ & & & & & & \\
\hline \hline-10 & 10 & -10 & -17.7778 & -10.7818 & -33.9972 & -10.0020 & 10.0038 & -10.0030 \\
0.8660 & 0 & 0.5000 & 0.9722 & 0.2300 & 0.0447 & 0.8659 & -0.0005 & 0.5001 \\
-1.0000 & 2.0000 & 1.7321 & -4.9816 & 20.7632 & 1.5182 & -0.9998 & 2.0003 & 1.7329 \\
\hline \hline
\end{tabular}

Table 3 - Example of reconstruction from very scarce data. Sensitivity was set at $S=4 \cdot 10^{-5}$, data is sampled from the grid $[-60,60] \times[-60,60]$, with spacing $h=10$. As previously $\lambda=\mu=1$. 

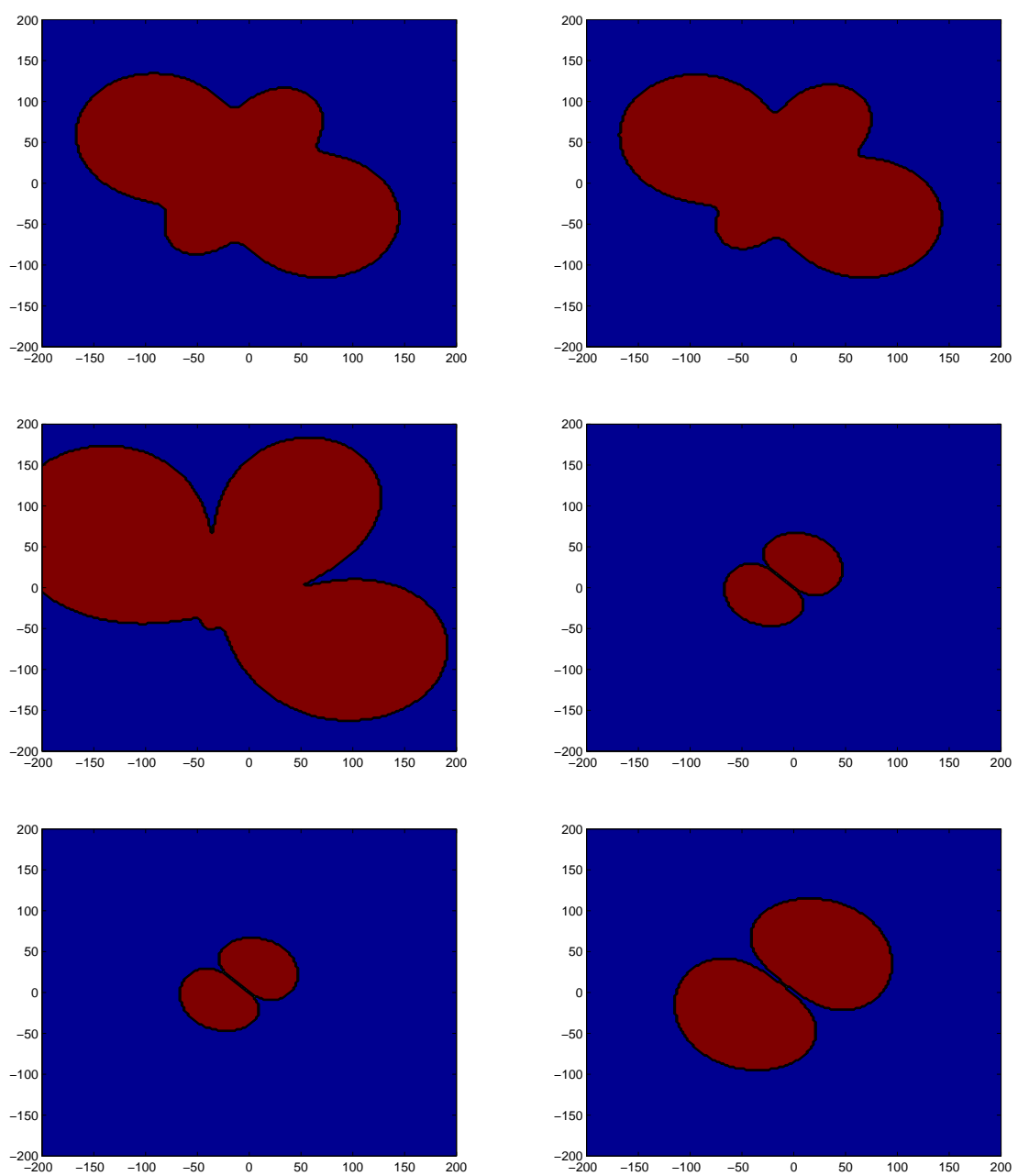

Figure 6 - The indicative sets corresponding to the surface data relative to the six cases reported in table 2. 

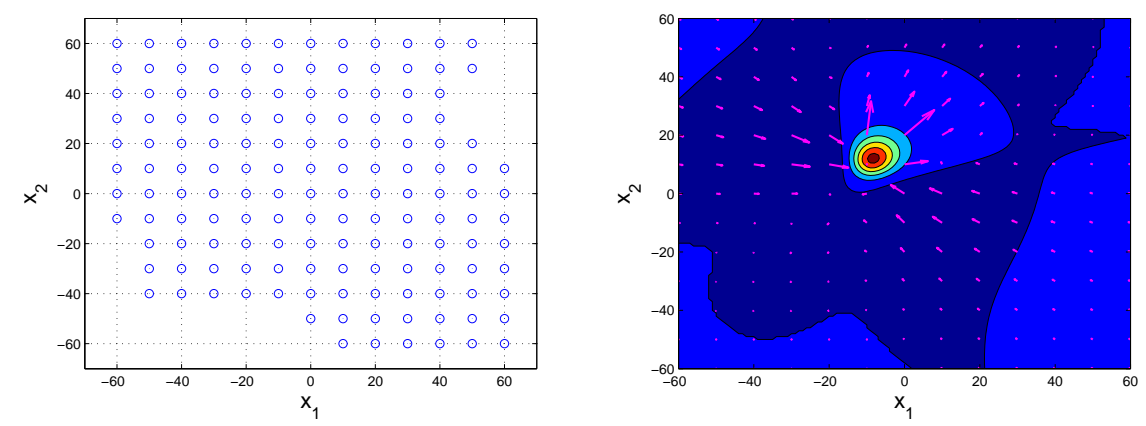

Figure 7 -Above: the set of data points used for the inversion procedure whose output is provided in table 3. Below: the corresponding surface field $u\left(x_{1}, x_{2}\right)$. 


\section{Appendix}

Instead of giving formulas for each entry of the matrix $H$, it is advantageous to write out formulas for the coordinates $H t$ where $t$ is the vector $\left(t_{1}, t_{2}, t_{3}\right)$. We only present in this appendix formulas at $x_{3}=0$ : the idea is to give a feel for the different terms involved. The complete formula for $x_{3}<0$ is best left within a computer code.

It proves convenient to introduce polar surface coordinates.

7.3. If $\left(x_{1}-y_{1}\right)^{2}+\left(x_{2}-y_{2}\right)^{2}=0$

The three coordinates of $H t$ are then, respectively,

$\begin{array}{r}0 \\ 0 \\ -\mu\left(n_{1} t_{1}+n_{2} t_{2}\right)+6(\lambda+\mu) n_{3} t_{3} \\ \hline 4 \pi y_{3}^{2}(\lambda+\mu)\end{array}$

7.4. If $\left(x_{1}-y_{1}\right)^{2}+\left(x_{2}-y_{2}\right)^{2}>0$

We set

$$
\begin{array}{r}
\rho=\sqrt{\left(x_{1}-y_{1}\right)^{2}+\left(x_{2}-y_{2}\right)^{2}} \\
d=\sqrt{\rho^{2}+y_{3}^{2}} \\
c=\frac{x_{1}-y_{1}}{\rho} \\
s=\frac{x_{2}-y_{2}}{\rho} .
\end{array}
$$

The first coordinate of $H t$ is the ratio of

$$
\alpha n_{1} t_{1}+\beta n_{2} t_{2}+\gamma n_{3} t_{3}+\delta\left(n_{1} t_{2}+n_{2} t_{1}\right)+\epsilon\left(n_{1} t_{3}+n_{3} t_{1}\right)+\zeta\left(n_{2} t_{3}+n_{3} t_{2}\right)
$$

to

$$
(\lambda+\mu) \pi \rho^{3} d^{5}
$$

where, setting

$$
A=\left(y_{3} d \rho^{4}+\left(5 / 2 y_{3}{ }^{4}+2 y_{3}{ }^{3} d\right) \rho^{2}+y_{3}{ }^{6}+y_{3}{ }^{5} d\right) \mu
$$

$\alpha, \beta, \gamma, \delta, \gamma, \epsilon, \zeta$ are given by,

$$
\begin{array}{r}
\frac{\alpha}{c}=-A\left(4 c^{2}-3\right)+ \\
\left(3 / 2 \lambda c^{2}+\mu\right) \rho^{6}-1 / 2 \mu\left(15 c^{2}-11\right) y_{3}^{2} \rho^{4}, \\
\frac{\beta}{c}=A\left(4 c^{2}-3\right)- \\
3 / 2 \lambda\left(c^{2}-1\right) \rho^{6}+3 / 2 \mu\left(5 c^{2}-4\right) y_{3}^{2} \rho^{4},
\end{array}
$$




$$
\begin{gathered}
\frac{\gamma}{c}=3 / 2(\lambda+\mu) y_{3}{ }^{2} \rho^{4}, \\
\frac{\delta}{s}=-A\left(4 c^{2}-1\right)+ \\
\left(3 / 2 \lambda c^{2}+1 / 2 \mu\right) \rho^{6}-1 / 2 \mu\left(15 c^{2}-4\right) y_{3}{ }^{2} \rho^{4}, \\
\epsilon=-3 / 2 \rho^{5} y_{3} c^{2}(\lambda+\mu), \\
\zeta=-3 / 2(\lambda+\mu) c s y_{3} \rho^{5} .
\end{gathered}
$$

The second coordinate of $H t$ is also in form of the ratio of (67) to (68), where this time $\alpha, \beta, \gamma, \delta, \gamma, \epsilon, \zeta$ are given by

$$
\begin{gathered}
\frac{\alpha}{s}=-A\left(4 c^{2}-1\right)+ \\
3 / 2 \lambda c^{2} \rho^{6}-3 / 2\left(5 c^{2}-1\right) \mu y_{3}{ }^{2} \rho^{4} \\
\frac{\beta}{s}=A\left(4 c^{2}-1\right)+ \\
\left(3 / 2 \lambda+\mu-3 / 2 \lambda c^{2}\right) \rho^{6}+1 / 2 \mu\left(-4+15 c^{2}\right) y_{3}^{2} \rho^{4} \\
\frac{\gamma}{s}=3 / 2(\lambda+\mu) y_{3}^{2} \rho^{4} \\
\frac{\delta}{c}=A\left(4 c^{2}-3\right)+ \\
\left(3 / 2 \lambda+1 / 2 \mu-3 / 2 \lambda c^{2}\right) \rho^{6}+1 / 2 \mu\left(15 c^{2}-11\right) y_{3}^{2} \rho^{4} \\
\epsilon=-3 / 2(\lambda+\mu) c s y_{3} \rho^{5} \\
\zeta=3 / 2 \rho^{5} y_{3}\left(c^{2}-1\right)(\lambda+\mu) .
\end{gathered}
$$

Setting,

$$
B=\left(1 / 2 d \rho^{5}+d y_{3}{ }^{2} \rho^{3}+\left(1 / 2 y_{3}{ }^{5}+1 / 2 y_{3}{ }^{4} d\right) \rho\right) \mu,
$$

the third coordinate of $H t$ is also in form of the ratio of (67) to (68), where this time $\alpha, \beta, \gamma, \delta, \gamma, \epsilon, \zeta$ are given by,

$$
\begin{gathered}
\alpha=-B\left(-1+2 c^{2}\right)+ \\
\left(-3 \mu c^{2}-3 / 2 \lambda c^{2}+\mu\right) y_{3} \rho^{5}-1 / 2 \mu\left(5 c^{2}-3\right) y_{3}{ }^{3} \rho^{3} \\
\beta=B\left(-1+2 c^{2}\right)+ \\
\left(-2 \mu+3 / 2 \lambda c^{2}-3 / 2 \lambda+3 \mu c^{2}\right) y_{3} \rho^{5}+1 / 2 \mu\left(-2+5 c^{2}\right) y_{3}{ }^{3} \rho^{3}
\end{gathered}
$$




$$
\begin{gathered}
\gamma=-3 / 2(\lambda+\mu) y_{3}{ }^{3} \rho^{3}, \\
\frac{\delta}{s c}=\left((-3 / 2 \lambda-3 \mu) y_{3}-d \mu\right) \rho^{5}+\left(-5 / 2 \mu y_{3}{ }^{3}-2 d \mu y_{3}{ }^{2}\right) \rho^{3}+\left(-d \mu y_{3}{ }^{4}-\mu y_{3}{ }^{5}\right) \rho, \\
\frac{\epsilon}{c}=3 / 2(\lambda+\mu) y_{3}{ }^{2} \rho^{4}, \\
\frac{\zeta}{s}=3 / 2(\lambda+\mu) y_{3}{ }^{2} \rho^{4} .
\end{gathered}
$$

Remark: Denote $\left(u_{1}, u_{2}, u_{3}\right)$ the coordinates of $H t$, whose expressions were given above. The following symmetry properties hold:

$$
\begin{aligned}
& u_{1}\left(s, c, n_{1}, n_{2}, t_{1}, t_{2}\right)=u_{2}\left(c, s, n_{2}, n_{1}, t_{2}, t_{1}\right), \\
& u_{3}\left(s, c, n_{1}, n_{2}, t_{1}, t_{2}\right)=u_{3}\left(c, s, n_{2}, n_{1}, t_{2}, t_{1}\right) .
\end{aligned}
$$

These symmetry properties can be easily verified using that $s^{2}+c^{2}=1$. Physically, they express that the first and the second coordinate play the same role for the displacement vector $H t$, on the surface $x_{3}=0$.

\section{ACKNOWLEDGMENTS}

This is joint work with I. R. Ionescu. Partial support for this work was provided by NSF grant DMS 0707421.

\section{REFERENCES}

[1] M. Abramowitz and I. Stegun, eds. (1992), Handbook of Mathematical Functions with Formulas, Graphs, and Mathematical Tables, Dover, New York.

[2] Dieterich, J.H. A model for the nucleation of earthquake slip, in Earthquake source mechanics, Geophys. Monogr. Ser., vol. 37, edited by S. Das, J. Boatwright, and C.H. Scholz, AGU, Washington, D. C. (1986), pp. 37-47.

[3] W.L. Elsworth and G.C. Beroza, Seismic evidence for an earthquake nucleation phase, Science, vol. 268 (1995), 851-855.

[4] Y. Iio, Slow initial phase of the P-wave velocity pulse generated by microearthquakes, Geophys. Res. Lett., vol. 19(5) (1992) pp. $477-480$.

[5] I. R. Ionescu, D. Volkov, An inverse problem for the recovery of active faults from surface observations, Inverse Problems 22 (2006) 2103-2121.

[6] I. R. Ionescu, D. Volkov, Earth surface effects on active faults: an eigenvalue asymptotic analysis, Journal of Computational and Applied Mathematics archive Volume 220, Issue 1-2 (October 2008).

[7] I. R. Ionescu, D. Volkov, Detecting tangential dislocations on planar faults from traction free surface observations, submitted to Inverse Problems.

[8] V. Kostoglodov, S. K. Singh, J.A. Santiago, S.I. Franco, K.M. Larson, A.R. Lowry and R. Bilham, A large silent earthquake in the Guerrero seismic gap, Mexico, Geophys. Res. Lett., Vol. 30 (15), (2003), doi:10.1029/2003GL017219

[9] A.R. Lowry, K.M. Larson, V. Kostoglodov and O. Sanchez, The fault slip budget in Guerrero, southern Mexico, Geophysical Journal, International, Vol. 200, (2005), pp. 1-15

[10] P. A. Martin, L. Päivärinta, S. Rempel, A normal crack in an elastic half-space with stress-free surface. (English summary, Math. Methods Appl. Sci. 16 (1993), no. 8, 563-579.

[11] R. D. Mindlin, Force at a point in the interior of a semi infinite solid, Physics Vol. 7, 1936, p 195-202.

[12] M. Ohnaka, Y. Kuwakara and K. Yamamoto, Constitutive relations between dynamic physical parameters near a tip of the propagation slip during stick-slip shear failure, Tectonophysics, 144 (1987), 109-125.

[13] V.Z. Parton, P.I. Perlin, Integral Equations in Elasticity, Moscow, Mir Publisher, 1982. MR 509209 (80a:73001)

[14] T. Sagiya and S. Ozawa, Anomalous transient and silent earthquakes along the Nankai Trough subduction zones, Seismol. Res. Lett., vol. 73 (2), (2002), 234-235

[15] Guang Y. Sheu, Deformations caused by the movements of shear and tensile faults, Int. J. Numer. Anal. Meth. Geomech., 2001, 25:1175-1193. 
[16] J. A. Steketee, On Volterra's dislocations in a semi infinite elastic medium, Canadian Journal of Physics, 36, 1958, p $192-205$.

[17] E. P. Stephan, A boundary integral equation method for three dimensional crack problems in elasticity, Mathematical Methods in the Applied Sciences, 1986, Volume 8-4, 609-623.

[18] D. Volkov, A double layer surface traction free Green's tensor, SIAM Journal of Applied Mathematics, in press. 